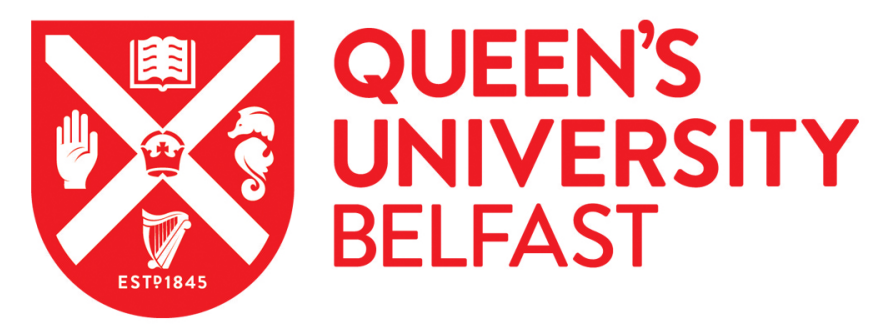

\title{
The Study of NF-kappaB Transcription Dynamics via Fluorescent Image Analysis
}

McArdle, A., McMenemy, K., \& Ferguson, S. (2008). The Study of NF-kappaB Transcription Dynamics via Fluorescent Image Analysis. 1-6. Paper presented at 8th IEEE International Conference on Biolnformatics and BioEngineering, Athens, Greece. https://doi.org/10.1109/BIBE.2008.4696802

Queen's University Belfast - Research Portal:

Link to publication record in Queen's University Belfast Research Portal

\section{General rights}

Copyright for the publications made accessible via the Queen's University Belfast Research Portal is retained by the author(s) and / or other copyright owners and it is a condition of accessing these publications that users recognise and abide by the legal requirements associated with these rights.

Take down policy

The Research Portal is Queen's institutional repository that provides access to Queen's research output. Every effort has been made to ensure that content in the Research Portal does not infringe any person's rights, or applicable UK laws. If you discover content in the Research Portal that you believe breaches copyright or violates any law, please contact openaccess@qub.ac.uk. 


\title{
The Study of Hepatic NF- $\kappa$ B Transcription Dynamics via Fluorescent Image Analysis
}

\author{
Alison McArdle, Karen McMenemy and Stuart Ferguson
}

\begin{abstract}
This paper presents an imaging technique for assessing the stimulation of $\mathrm{H35}$ cells by TNF- $\alpha$. Specifically, fluorescence from the stimulated cells is measured in order to produce intensity profiles that describe the dynamics of the NF- $\kappa$ B transcription factor. The approach offers a costeffective, non-invasive and quantifiable alternative to traditional techniques used to acquire this intracellular information. The analysis successfully extracts information from low contrast images and produces profiles comparable to those published in previous literature. The images are de-noised using wavelets, automatic thresholding is performed and connected component analysis is carried out, so that it is possible to track fluorescent cell clusters in a series of images taken at a high sample rate. The resulting profiles showed that the level of $N F-\kappa B$ in the cells increased as the concentration of stimulating TNF- $\alpha$ increased. They also provided supporting evidence for the hypothesis that the time of maximum NF- $\kappa \mathrm{B}$ response does not depend on the initial stimulating TNF- $\alpha$ concentration. Experimental results such as these are crucial for the development and validation of mathematical models describing intracellular signal transduction pathways.
\end{abstract}

\section{INTRODUCTION}

$\mathrm{NF}-\kappa \mathrm{B}$ transcription factors have attracted a high level of interest from scientists due to their ability to regulate a vast number of genes associated with inflammatory responses and other stress conditions. They have been identified as possible drug targets for chronic inflammatory diseases and also play a key role in tissue repair [1], [2].

Characterising the expression dynamics of NF- $\kappa \mathrm{B}$ is a necessary step in realising its full potential. Typical techniques for acquiring this data, such as Western Blot Analysis and Fluorescence Activated Cell Sorting (FACS), are limited in their approach as they involve disturbing the sample, provide semi-quantitative data and in the case of FACS require costly laboratory equipment [3], [4]. This paper describes a quantifiable, cost-effective and readily available image analysis approach for acquiring profiles from image data to describe

Manuscript received July 4, 2008. This work was supported by a DEL Postgraduate Research Studentship.

Alison McArdle is with the School of Electronics, Electrical Engineering and Computer Science, Queen's University Belfast, United Kingdom (phone: +44(0)28 9097 4164; fax: +44(0)28 9067 7023; e-mail: amcardle02@qub.ac.uk)

Karen McMenemy is with the School of Electronics, Electrical Engineering and Computer Science, Queen's University Belfast, United Kingdom (e-mail: k.mcmenemy@ee.qub.ac.uk)

Stuart Ferguson is with the School of Electronics, Electrical Engineering and Computer Science, Queen's University Belfast, United Kingdom (e-mail: r.ferguson@ee.qub.ac.uk) gene dynamics, comparable to those found by traditional techniques, with the added advantage of being a non-invasive measurement.

The images analysed in this paper, were captured at regular intervals of $\mathrm{H} 35$ cells that were transfected with a green fluorescent protein reporter cell line and stimulated by a comprehensive range of tumor necrosis factor (TNF- $\alpha$ ) concentrations. TNF- $\alpha$ is a cytokine, recognised as one of the main mediators of the acute phase response (APR), which is a beneficial mammalian response to tissue trauma, injury and infection [5]. As the concentration of TNF- $\alpha$ is increased, the levels of fluorescence in the cells vary and this change in fluorescence corresponds to the changing concentration of $\mathrm{NF}-\kappa \mathrm{B}$ in the cells. The results from this experiment will be used to aid in the development of a mathematical model describing intracellular signal transduction in hepatocytes when stimulated by TNF- $\alpha$.

The image analysis described in this paper involves contrast boosting, de-noising using wavelets and automatic thresholding to identify fluorescing regions. Wavelet analysis techniques, although relatively new mathematical tools, are well utilised; for example, they are the chosen method of the Federal Bureau of Investigation (FBI) for the compression of fingerprints [6]. They are also highly successful in their application to de-noise images through their ability to shift and scale versions of a chosen mother wavelet to accurately decompose and then reconstruct the original image, while eliminating both low and high frequency noise [7].

Upon identification of the relevant regions in each image, connected component analysis is performed which labels each detected cell or fluorescent region, calculates parameters relating to each region and allows for the possibility of tracking these objects through a series of images taken at a higher sample rate, using algorithms such as the KanadeLucas-Tomasi feature tracker (KLT) [8]. In this preliminary work, we do not track individual cells between the images, however it is an area of future work identified in Section V. The work described here concentrates on producing intensity profiles for the series of images collected.

In order to arrive at the final set of intensity profiles for each experimental condition it is necessary to correct for fluctuations in background intensity. This requirement is confirmed through a control experiment. This is discussed in more detail in Section IV.

Previous work on similar images of H35 cells involved 
an analysis using FACS, principal component analysis and alternative algorithms to detect the fluorescing regions in the images [9], [10]. The results produced in this work compare well to the profiles found through these alternative methods, but the method described here uses pre-existing techniques which are readily available and permits for tracking the objects if the images are taken at a high enough frequency.

Other work involving the analysis of fluorescent images on different cell types, has been heavily focused on dealing with well-defined objects and the analysis of single molecules [11], [12].

Section II of the paper introduces the experimental setup and image acquisition procedures. This is followed (in Section III) by a detailed description of the steps involved in the analysis of the images. Section IV presents the results and provides a comparison with the published work of others. A concluding summary is detailed in Section V and is followed by important acknowledgements in Section VI.

\section{NF- $\kappa$ B IMAGE ACQUISITION}

H35 cells were transfected with a DNA plasmid encoding green fluorescent protein (GFP) which results in the emission of a green fluorescence upon the transcription of NF- $\kappa \mathrm{B}$ [13].

An array was setup consisting of 16 samples treated with 8 different experimental conditions, thereby having two samples for each condition. The range of conditions correspond to different stimulating concentrations of the cytokine TNF- $\alpha$ and are detailed in Table I.

TABLE I: Experimental Conditions

\begin{tabular}{c|c} 
Sample & Stimulating Concentration of TNF- $\alpha$ \\
\hline \hline 1,9 & $25 \mathrm{ng} / \mathrm{ml}$ \\
2,10 & $22 \mathrm{ng} / \mathrm{ml}$ \\
3,11 & $19 \mathrm{ng} / \mathrm{ml}$ \\
4,12 & $17 \mathrm{ng} / \mathrm{ml}$ \\
5,13 & $13 \mathrm{ng} / \mathrm{ml}$ \\
6,14 & $9 \mathrm{ng} / \mathrm{ml}$ \\
7,15 & $6 \mathrm{ng} / \mathrm{ml}$ \\
8,16 & $0 \mathrm{ng} / \mathrm{ml}($ Control $)$
\end{tabular}

A Zeiss 200 Axiovert microscope was used to capture fluorescent images using an AxioCAM MRM digital camera with an exposure time of $64.87 \mathrm{~ms}$ and an image size of 1388 x 1040 pixels. Twenty-two images were captured at one hour intervals, with the first image acquired 1 hour after the initial activation by the stimulant.

Images were presented in the ZVI format which was developed by Carl Zeiss to ensure that each file contains not only the image but also the important acquisition and measurement settings.

Each image consists of information from two different channels. The first channel is a visual image of the cells and their microscopic features, and the second channel is from a standard FITC bandpass filter and is used to determine the green fluorescent intensity. By converting these ZVI files to individual bitmaps and subdividing them into their separate channels, the images associated with the FITC channel could be isolated and used for the analysis to follow.

\section{IMAGE ANALYSIS}

This section describes the procedure used to extract the relevant information from the collected images. All steps have been carried out using MATLAB.

\section{A. Image Formatting}

The images are cropped to eliminate artifacts which distort the boundary regions. They are then converted from 8 bit per channel RGB colour space to a greyscale intensity, in the range of $0-1$. This double precision format allows for greater accuracy in calculations. Only the green $(\mathrm{G})$ component from the fluorescing images is used to determine the greyscale since the red (R) and blue (B) components are negligible.

The original images have such low level contrast that they appear as black to the human eye. In order to boost the contrast the greyscale intensity values are mapped to new values by stretching the upper and lower pixel values so that $1 \%$ of the data is saturated at both limits. Figure 1 shows the affect of this transformation for the first image taken of sample 1 . Clearly the boost in contrast helps to visually identify regions of fluorescing cells. Since the contrast boosting that is applied to each image is dependent upon the upper and lower pixel grey levels in that image, it is not possible to apply the same contrast boost to each image. However, the boost in contrast is only used to create a mask that can be applied to the original image in order to identify fluorescing regions. This part of the analysis is detailed further in Section III, part C.

\section{B. Wavelet De-noising}

After boosting the contrast of the image, noise was clearly evident. In order to remove this, a wavelet analysis was performed. Wavelets decompose the signal, which in this case is an image, using both low and high pass filters and then reconstruct them using another set of low and high pass filters. A 3rd order coiflet wavelet was chosen for this application for its effectiveness, possibly associated to the likeness between the mother wavelet and the typical changes in intensity found in any given cross section of the images. Figure 2 shows such a cross section of an image of Sample 1 taken 10 hours after the experiment began and also shows a profile of the mother wavelet for the third order coiflet used in this analysis. The structure of the 4 filters associated with a 3rd order coiflet are presented in figure 3 .

This 3rd order coiflet is applied at a decomposition level of 4 , using automatically determined values for soft thresholding. These values are obtained for each image using the wavelet coefficients and the Birgé-Massart penalisation method. This method has been described in literature as a 

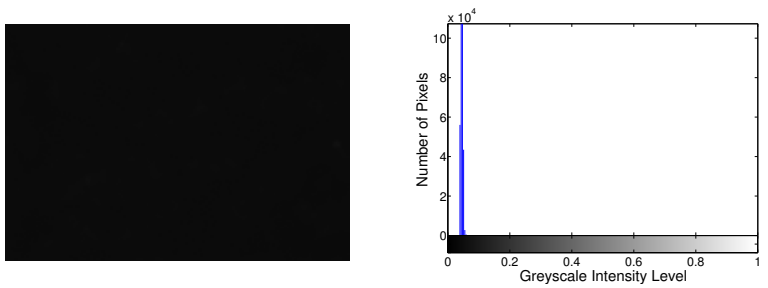

(a)
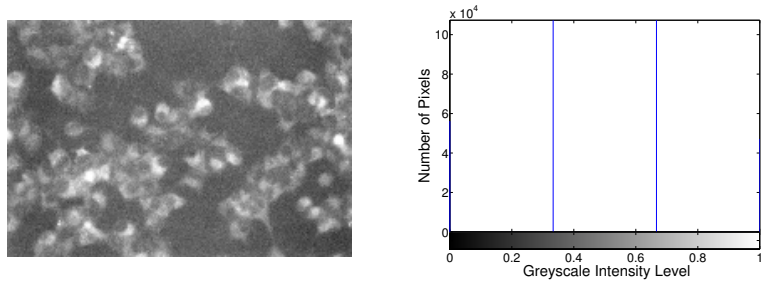

(b)
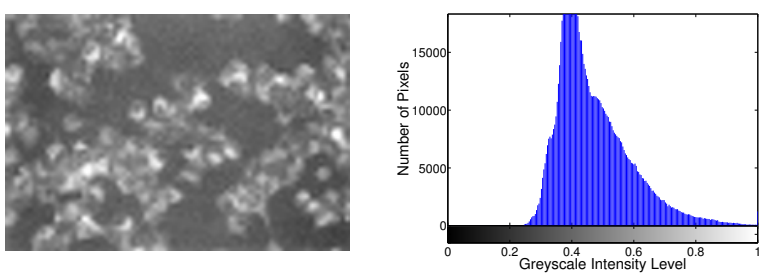

(c)

Fig. 1: Analysis of image taken of Sample 1 after 1 hour of stimulation by $25 \mathrm{ng} / \mathrm{ml}$ of TNF- $\alpha$. Image and associated histogram (a) prior to contrast boosting, (b) after contrast boosting, and (c) after wavelet de-noising.

highly flexible thresholding estimator which retains a greater number of coefficients at higher levels of decomposition, as are the conditions in this wavelet analysis [14]. Figure 1 shows the resulting image and associated histogram after the de-noising has been performed.

\section{Identifying Fluorescing Cells}

With the images successfully de-noised, the next step is to identify which areas of the images contain fluorescing cells. A visual inspection of both the images and their associated histograms of pixel distribution would allow for the selection of a reasonable threshold to extract the fluorescing pixels from the background. However, there is an obvious degree of estimation in this method. With the added visual evidence that there are fluctuations in the background lighting conditions throughout the full set of the original 22 images, it was decided that an automatic thresholding would be the most appropriate technique to use. This variation in background lighting will be discussed in detail in Section IV.

Otsu's method is used to automatically choose the threshold value for each image. This method chooses an optimum threshold by separating pixels into two classes, which would be background and foreground in this case, and then minimising their within-class variance [15].

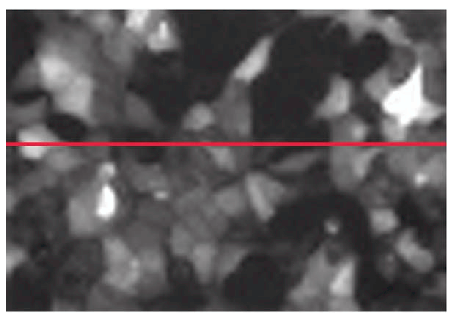

(a)

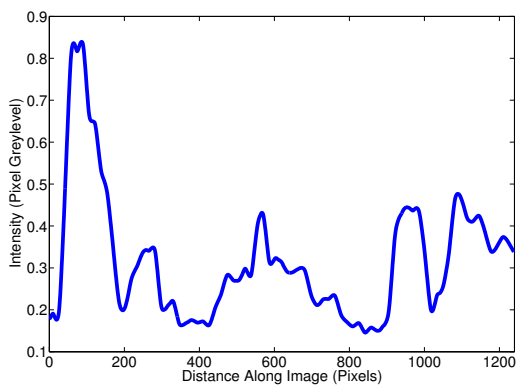

(b)
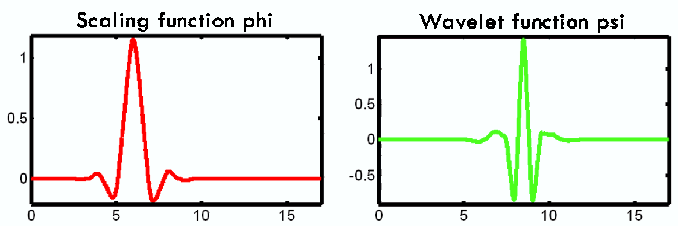

(c)

Fig. 2: Figure showing the similarity between the intensity profile across a sample image and that associated with the mother wavelet for the coiflet. (a) Image taken after 10 hours of Sample 1 with a red line indicating the cross section analysed for its intensity profile, (b) Intensity profile along the cross section marked in the above image and (c) Mother wavelet associated with 3rd order coiflet.

This threshold is then used to produce a binary image which acts as a mask on the original greyscale image, eliminating the background and allowing through only the fluorescing pixels. Figure 4 shows how this binary image is used to mask out the background pixels and thereby show only those areas detected as fluorescing.

Connected component analysis is used to identify fluorescing regions, with a minimum of 8 connected components constituting a cell. This step helps eliminate any fluorescing pixels which may be due to noise as opposed to part of the cells of interest. For this application it was found the results did not offer any further elimination of the number of detected fluorescing pixels, and hence every fluorescing region can be considered, with added confidence, to be representative of a cell. Furthermore, each identified cell is labelled and their centre of mass calculated so as all parameters are in place to permit the use of a tracking algorithm. However, tracking is not feasible with the set of images analysed in this work as they have not been acquired at a high enough sample rate. 

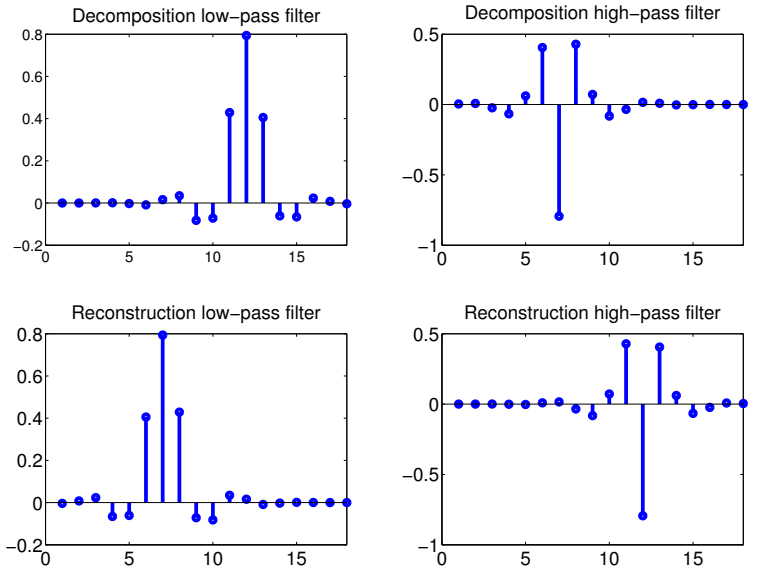

Fig. 3: Low and high pass filters which compose the 3rd order coiflet wavelet.

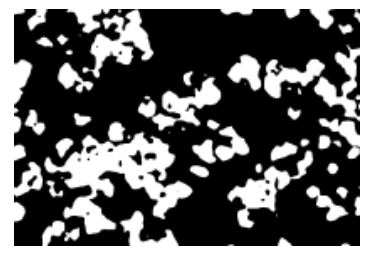

(a)

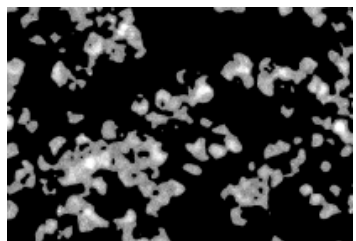

(b)
Fig. 4: Analysis of image taken of Sample 1 after 1 hour of stimulation by $25 \mathrm{ng} / \mathrm{ml}$ of TNF- $\alpha$. (a) Binary image produced after thresholding and connected component analysis, (b) image produced when binary image is used as a mask on original greyscale image.

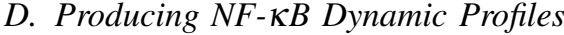

Since a variation in the lighting conditions was found from image to image, even in the control experiment as shown in figure $5 \mathrm{~b}$, this suggests that the fluctuations are not due to relevant biological changes in the cell samples. It is therefore necessary to correct for this change so that the intensity values calculated for each image are comparable over time.

Therefore the intensity of the identified fluorescing pixels and the eliminated background pixels by the masking binary image, respectively, are averaged. The average background intensity value is then subtracted from the average fluorescence intensity to correct for the variation in the lighting conditions. This was repeated for the images acquired at each time point, and an intensity profile produced. Thus, this newly generated intensity profile can be assumed to be caused by only the fluorescing cells.

Since there were two sets of experiments for each stimulating concentration of TNF- $\alpha$, the matching intensity profiles were averaged to produce a final intensity profile for each experimental condition. The values are then multiplied by 255 to change from the $0-1$ scale back to the original grey level range $0-255$. Since only the $\mathrm{G}$ value was used originally to produce the intensity image, the NTSC conversion back to each original RGB component was not necessary.

\section{PRESENTATION OF RESULTS}

This sections presents the intensity profiles acquired via the analysis of the fluorescent images and compares these results to previously published findings.

\section{A. Intensity Profiles}

Figure 5a shows the intensity profiles of the fluorescing regions only (prior to subtraction of the background intensity), for each of the seven different stimulating concentrations of TNF- $\alpha$ and figure $5 b$ shows the profile of how the background intensity varies with time. It is clear that there is minimal difference between what we classify as the bright areas of the image caused by fluorescence and the dark areas. This is in the order of two pixel grey levels. It is also evident that the intensity profile for the background pixels follows a similar trend for each experimental condition, including the control condition where no stimulus has been added. This may be due to changing environmental and lighting conditions over the duration of the 22 hour experiment or the possibility that camera parameters, such as the white balance, were not held static throughout.
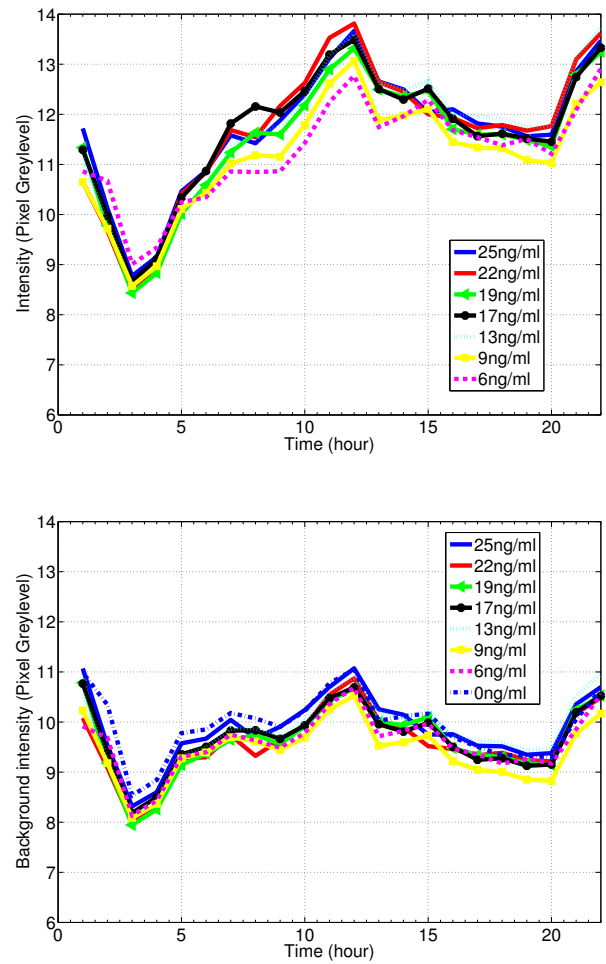

(b)

Fig. 5: Comparison of intensity profiles of (a) fluorescing pixels, and (b) background pixels, for the different stimulating concentrations of TNF- $\alpha$.

The intensity profile for the control experiment is not displayed for the bright pixels since the images were distorted by an artifact. Since this artifact was successfully removed after thresholding, the average background intensity could still be accurately acquired. Figure 6 shows the first image 
taken of the control experiment which shows the appearance of the artifact (ring like feature) as the image goes through the stages of de-noising, thresholding and finally masking the bright pixels, to leave the useful information encapsulated in the background pixels.

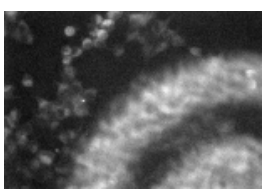

(a)

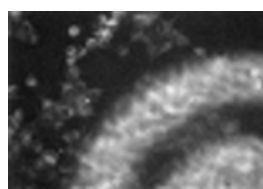

(b)

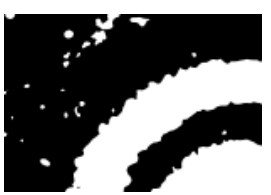

(c)

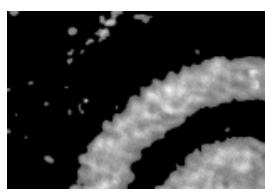

(d)

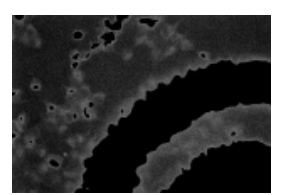

(e)
Fig. 6: Control image taken of Sample 8 after 1 hour. (a) Greyscale image, (b) denoised image, (c) thresholded binary image, (d) masking out dark pixels of original image to show fluorescing regions only, (e) masking out fluorescing regions of original image to show background pixels only.

The background intensity values are then subtracted from the foreground intensity values to produce the final intensity profiles for each of the experimental conditions as shown in figure 7. The level of NF- $\kappa \mathrm{B}$ generally shows an increase with increasing concentration of TNF- $\alpha$, while its dynamic behaviour shows little or no variation throughout the different experimental conditions.

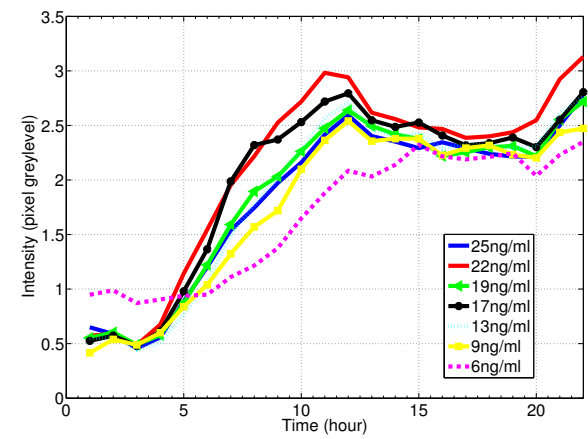

Fig. 7: Comparison of final intensity profiles produced for each stimulating concentration of TNF- $\alpha$.

\section{B. Comparison with Previous Results}

King et al. have carried out research on the analysis of the transcription factor NF- $\kappa \mathrm{B}$. Although the same $\mathrm{H} 35$ cells were used in their analysis, the only stimulating concentration of TNF- $\alpha$ documented is that of $25 \mathrm{ng} / \mathrm{ml}$ [9].

An approximation of the results found by King et al., using FACS and an alternative analysis of fluorescent microscopy images, have been used in figure 8 as a comparison to the profile acquired in this work for the same stimulating concentration of TNF- $\alpha$.
The profile found through the analysis described in this paper was normalised using two different methods so that it would be comparable to other results. The first normalisation was performed by scaling all values by the same amount. In this case, the normalisation factor was considered to be the value at which the profile appeared to reach a steady state (depicted in the timeline from 11-16 hours). Typically a maximum value might normally be used to achieve normalisation. However, in the case of the $25 \mathrm{ng} / \mathrm{ml}$ stimulation, the actual maximum intensity occurred at $22 \mathrm{hrs}$. This is considered to be an anomaly, since biologically maximum activity is generally reached after 10 hours. Thus it was thought better to use the steady state value for normalisation.

The second normalisation was performed using a similar calculation to that described by King et al. which is detailed in (1).

$$
I_{N}(t)=\frac{\left[I(t)-I_{\min }\right]}{\left[I_{\max }-I_{\min }\right]}
$$

where, $I_{N}(t)$ is the normalised intensity at time $t, I(t)$ is the fluorescent intensity prior to normalisation and $I_{\min }$ and $I_{\max }$ represent the minimum and maximum fluorescent intensity respectively.

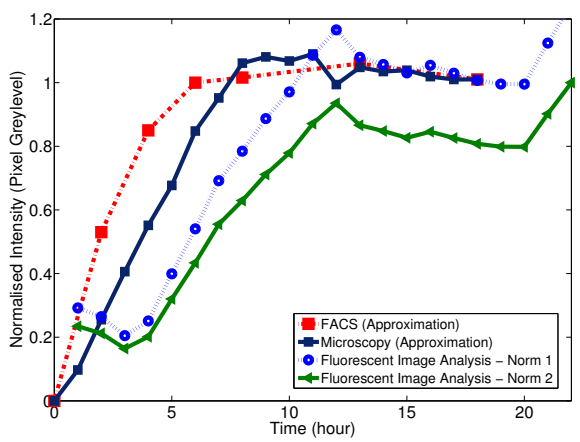

Fig. 8: Comparison of normalised intensity profiles for representing the dynamics of NF- $\kappa$ B transcription in $\mathrm{H} 35$ cells after stimulation by $25 \mathrm{ng} / \mathrm{ml}$ of TNF- $\alpha$. Approximated plots are shown for the results found via FACS and Microscopy from work by King et al. Two alternative sets of normalised results are shown for the dynamic profiles obtained from the analysis described in this paper.

\section{Discussion}

Regardless of the normalisation method used, it can be seen that the profiles produced using our algorithm are in strong agreement with those produced by King et al. The major anomalies appear both at the very start and at the very end of the profile (the first two data points and the final data point). In all cases, a higher intensity than expected has been found. There does not appear to be any biological reason for this. Since this trend appears for all concentration samples, at the same time points, the only conclusion that can be drawn is that some change in experimental conditions has occurred at these time points. This may have been changing illumination levels in the environment, or perhaps 
even changing camera parameters such as an adjustment to the automatic white balance. In addition, as previously noted, the difference between what is considered a bright and a dark pixel is as little as two integer steps in grey levels. As such, the selection of an appropriate threshold is critical to the overall determination of intensity levels. Therefore, it might be prudent to consider more intuitive methods of threshold determination. However both normalised profiles do show that the maximum value of NF- $\kappa \mathrm{B}$ for each experiment occurred in the region of 10-12 hours after initial stimulation by TNF- $\alpha$ and generally increased with increasing stimulation concentration. Biologically this is what we would expect to occur. The transient form of the response (including the time of maximum response) however did not seem to change as the stimulating concentration changed. This observation has also been noted previously in the literature, [9]. As a proof of concept, this initial research has shown it is possible to use simple imaging techniques to rapidly extract important information regarding cell biology from collected image samples.

\section{CONCLUSIONS AND FUTURE WORK}

\section{A. Conclusions}

A new approach to acquiring transient profiles for gene expression has been presented using image analysis techniques. The procedure not only has an advantage over traditional methods, in that it is cost-effective, non-invasive to the cell sample and provides quantifiable measurements, but also because of its apparent success at extracting relevant information for low contrast images that appear as black to the human eye.

A coiflet wavelet was successfully used to de-noise the images and Otsu's method applied for automatic threshold selection. The binary images produced were effective in acting as a mask when calculating the average intensity of background and foreground pixels. This proved particularly useful in the removal of the artifact in the control experiment images so as the variation in background lighting could still be detected.

The profiles produced are in strong agreement with those previously found via the traditional methods. The maximum value of NF- $\kappa \mathrm{B}$ for each experiment occurred in the region of 10-12 hours after initial stimulation by TNF- $\alpha$ and generally increased with increasing stimulation concentration.

When carrying out experiments involving the capture of images, it is crucial to keep the surrounding environment and camera settings as static as possible. The automatic white balancing on the camera should be deactivated, as this feature would otherwise continue to modify the white reference in the camera with changing lighting conditions and as a result, all subsequent calculations would be effected, and possibly result in changing background fluorescence as experienced in the images analysed in this work.

\section{B. Future Work}

Future work will be carried out into developing a more advanced thresholding analysis to help detect greater levels of information from the images. If a set of images were made available with a higher sample rate, then this algorithm, since connected component analysis has been used to label regions within an image, should then lend itself well to tracking the cells through images and providing intensity profiles at a cellular level.

\section{ACKNOWLEDGMENTS}

The authors gratefully acknowledge the contribution of Dr. Juergen Hahn, Zuyi Huang, Dr Arul Jayaraman and Fatih Senocak from Texas A\&M University, U.S.A, for performing the experimental work and kindly contributing the images for this analysis.

\section{REFERENCES}

[1] S. Ali and D.A. Mann, Signal Transduction Via The NF- $\kappa$ B Pathway:A Targeted Treatment Modality for Infection, Inflammation and Repair, Cell Biochemistry and Function, vol. 22, 2004, pp 67-79.

[2] Q. Li and I.M. Verma, NF- $\kappa$ B Regulation In The Immune System, Nature Reviews Immunology, vol. 2, 2002, pp 725-734.

[3] T. Lipniacki, P. Paszek, A.R. Brasier, B. Luxon and M. Kimmel, Mathematical Model of NF- $\kappa$ B Regulatory Module, Jour. of Theoretical Biology, vol. 228, 2004, pp 195-215.

[4] S. Subramanian and F. Srienc, Quantitartive Analysis of Transient Gene Expression in Mammalian Cells Using the Green

[5] H. Baumann and J. Gauldie, The Acute Phase Response, Immunology Today, vol.15, 1994, pp 74-80.

[6] J.N. Bradley, C.M. Brislawn and T. Hopper, FBI wavelet/scalar quantization standard for gray-scale fingerprint image compression, Proc. SPIE, vol. 1961 (293), 1993, pp 293-304.

[7] I. Daubechies, Ten Lectures on Wavelets, Society for Industrial and Applied Mathematics (SIAM), Philadelphia, PA; 1992.

[8] C. Tomasi and T. Kanade, Detection and Tracking of Point Features, Shape and Motion from Image Streams: A Factorization Method-Part 3, Technical Report CMU-CS-91-132, April 1991.

[9] K.R. King, S. Wang, D. Irimia, A. Jayaraman, M. Toner and M.L. Yarmush, A High-Throughput Microfluidic Real-Time Gene Expression Living Cell Array, Lab on a Chip, vol. 7, 2007, pp 77-85.

[10] Z. Huang and J. Hahn, Development and Comparison of Algorithms for Analysis of Fluorescent Images for Studying The Dynamics of Signal Transductions Pathways, AICHE, Annual Meeting, Salt Lake City, Utah, 2007.

[11] S. Venkataraman, Automated Image Analysis of Fluorescence Microscopic Images to Identify Protein-Protein Interactions, Proceedings of the IEEE Engineering in Medicine and Biology 27th Annual Conference, Shanghai, China, 2005, pp 4437-4440.

[12] A. Furtado and R. Henry, Measurement of Green Fluorescent Protein Concentration in Single Cells by Image Analysis, Analytical Biochemistry, vol. 310, 2002, pp 84-92.

[13] K.J. Weider, K.R. King, D.M. Thompson, C. Zia, M.L. Yarmush and A. Jayaraman, Optimization of Reporter Cells for Expression Profiling in a Microfluidic Device, Biomedical Microdevices, vol. 7 (3), 2005, pp 213-222.

[14] Y. Feng, S. Thanagasundram and F.S. Schlindwein, Discrete WaveletBased Thresholding Study on Acoustic Emmission Signals to Detect Bearing Defect on a Rotating Machine, The Thirteenth International Congress on Sound and Vibration, Vienna, Austria, July, 2006.

[15] N. Otsu, A Threshold Selection Method from Gray-Level Histograms, IEEE Transactions on Systems, Man and Cybernetics, vol. SMC-9, No. 1, 1979, pp 62-66. 